Reprinted from SCIENCE, 303, 778-779 (2004)

\title{
Pas de Deux for Atomic Electrons
}

\author{
C. R. Stroud, Jr.
}

An atom with many electrons is like a complex ballet, displaying an often chaotic interplay of orbits. Owing to the effects of quantum mechanics, the lowest energy orbits (or ground states) of many electron atoms are stable. Excited states of the atoms do experience interactions, however, in which one of the electrons is liberated while the other falls to a lower state. The connection between these autoionization processes and the classical orbits is generally obscured by these same quantum effects that stabilize the ground states, but in an experiment reported by Pisharody and Jones on page 813 of this issue, the connection is greatly clarified(1).

In classical mechanics, a multi-electron atom is very similar to a planetary system [for a nice description of the classical three-body problem, see (2)]. There are some differences, particularly the fact that the gravitational forces coupling planets with each other and with the Sun are always attractive while the electrons all are attracted to the nucleus, but repel each other. The classical orbits of the two systems have many similarities including the presence of chaos, and collisions between planets or electrons which can throw them free of their respective systems. Of course, we observe in nature that our solar system is relatively stable, and furthermore the ground states of multielectron atoms are stable. The reasons for this stability are different in the two cases. In the solar system the planets are in nearly circular orbits, each with a different radius, so that they are never very near each other. Their gravitational force on each other is proportional to the product of the masses, and since their masses are much smaller than that of the sun, the coupling with the sun is much stronger than the planets' mutual attraction unless they get very close together indeed. Were the planets to have very eccentric orbits so that they crossed then the probability of a close collision would be much greater and the planetary system would be unstable (3).

In contrast, the ground state atomic electrons are in highly eccentric low angular momentum orbits that cross and also venture near the nucleus where their kinetic energy is very large, allowing large exchanges of kinetic energy in collisions. It is rather surprising then that the atoms are stable. The classical description of the simplest multielectron atom, helium, was extensively investigated at the beginning of the last century in an attempt to apply the old Bohr model (4). The attempt failed and ultimately led to the development of modern quantum theory. In modern quantum theory the smearing effects mentioned earlier lead to the stability of the ground state. Excited states of the twoelectron atom in which each electron is in a bound state, but the sum of the energies of the two excited electrons is larger than the binding of a single electron are observed to be 
unstable undergoing autoionization in which one electron takes the energy of both electrons and is ejected while the second electron drops back to the ion ground state.

Can the instability of these excited states be tied back to correlated motion of the two electrons in classical orbits? Or does quantum mechanics completely smear the state of the electrons so that all remnants of the classical motion is lost? There has been significant progress towards an answer in the past few years. First, there has been progress on the theoretical side in developing a semiclassical treatment of helium based on a summation over classical orbits.[for a review, see (5)]. Second, there have been a series of calculations and experiments studying the ionization of helium and other atoms with two valance electrons subject to a very short intense laser pulse (6-9). Those studies have investigated whether the two electrons are ejected sequentially as each in its turn absorbs energy from the laser pulse, or are they ejected simultaneously by being excited and then colliding. Such studies are complicated by the fact that such a single intense laser pulse does not generally excite a single type of electron orbit.

Over the past 20 years, researchers in a number of laboratories have demonstrated that by use of a series of tailored short laser and $\mathrm{THz}$ pulses atomic electrons in single electron atoms can be excited into states in which a few or even a single electron orbit is excited and the electrons travel as localized wave packets along classical trajectories (10-14). In these studies it was found that the electron wave packets undergo a complex evolution of oscillations, decays, revivals, and fractional revivals in a sort of elaborate dance around the classical orbit.

This form of excitation is clearly ideal for studying the nature of correlations in multielectron atoms, but it has been slow in occurring because it requires synchronizing a whole train of picosecond laser pulses of different frequencies and durations to excite and probe the atoms. There has been some progress in experiments in which one electron was excited into an eigenstate or coherent mixture of two eigenstates and the second in a localized wave packet state $(15,16)$, but until the paper by Pisharody and Jones no one had reported an experiment in which the correlation of the motion of two atomic electrons in their orbit was directly controlled and measured.

The experiment was carried out using a barium atom. The two valance electrons were excited by a sequence of four picosecond laser pulses, with two used to excite each electron (see the figure). The pulses were timed so that the first pair excited one electron into a state which was made up of a superposition of elliptical orbits all of the same eccentricity and phase, but with various angular orientations. The result is a radially localized wave packet oscillating in and out at the classical orbital frequency. The second pair of pulses is then sent in after a variable delay and excited into another similar set of orbits making up a radially localized wave packet. The excited wave packet states are indicated schematically in the cutaway portion of the figure. The delay between the pulse trains exciting the two wave packets determines the relative phase of the motions of the two electrons in their orbits, and thus when they are both localized near the nucleus in such a way that they can collide in such a way as to liberate one of the electrons. After 
the process is complete Pisharody and Jones were able to analyze the remnant ion and electron and determine the nature of the collision.

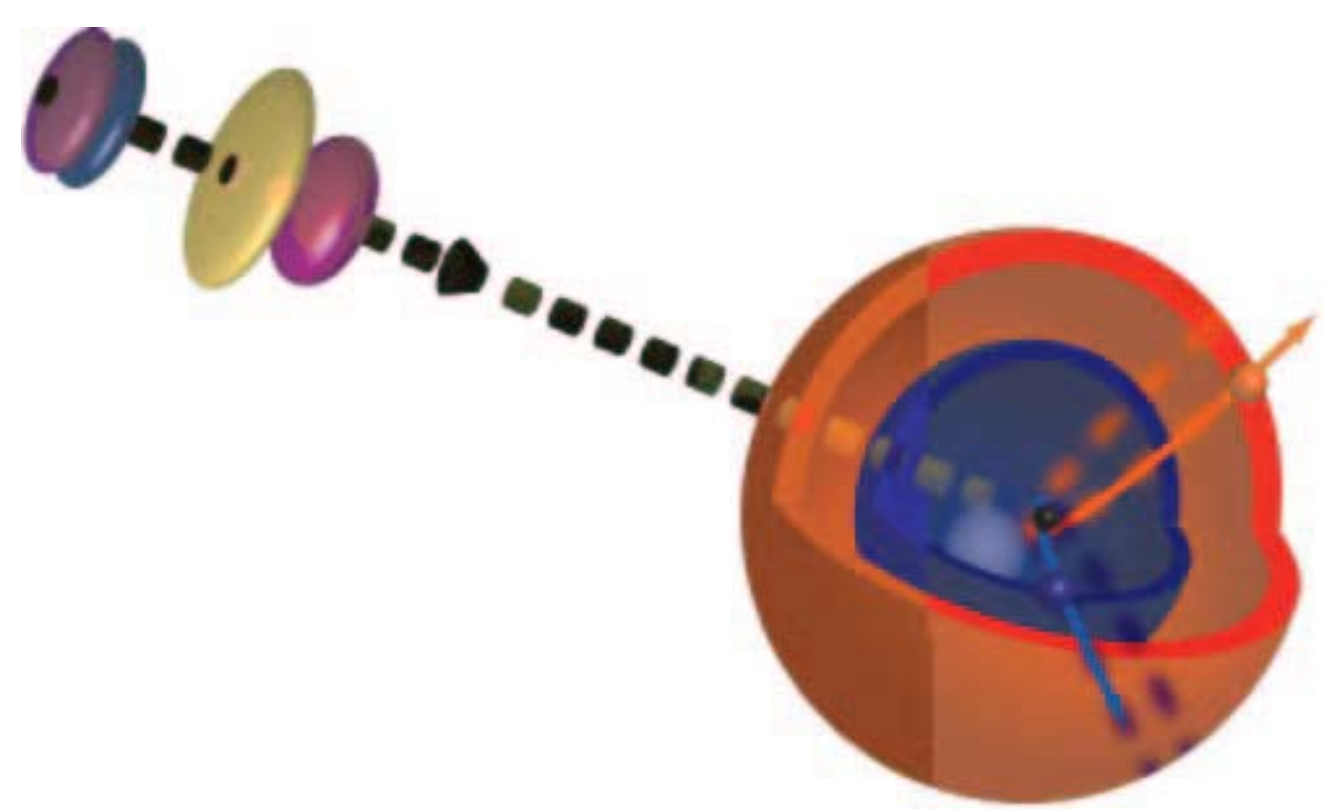

Dance of the electrons. In the experiment by Pisharody and Jones (1), a sequence of four laser pulses excites the two valance electrons into radial wave packets whose corresponding classical orbits are indicated. The outer electron (orange) is shown both in terms of one of its classical obits and in terms of its radial wave packet. The electron excited into the lower energy orbit (blue) is shown in one of its classical orbits. Varying the timing of the pulses allows control of the collision dynamics of the two electrons.

Van Vleck (17) showed us many years ago that the propagation of a quantum mechanical state could be described in terms of a superposition over all possible paths from an initial to a final state, so it is not surprising that the classical orbits underlie the quantum evolution of the excited two-electron atom, what is remarkable is that it is possible to use a complex train of precisely trained laser pulses to choreograph the motion of the two electrons in an elaborate pas-de-deux. It opens a whole new era in which the correlations and entanglements of multi-electron atoms can be explored and manipulated to finally bring to light the complicated electronic dance that is obscured when we limit ourselves to study of only atomic energy eigenstates. Many surprising types of classical orbits are already known from numerical simulations, orbits with both electrons on the same side of the nucleus, non-spreading wave packets, orbits in which one electron sits still in space 18-19). We should soon see these in the laboratory.

\section{References and Notes}

1. S. N. Pisharody, R. R. Jones, Science 303, 813 (2004). 
2. M. C.Gutzwiller, Rev. Mod. Phys. 70, 589 (1998).

3. The orbits of Pluto and Neptune do indeed cross, but two related factors combine to prevent a destabilizing collision between them. Their orbits are extremely large, making a near collision relatively less likely, and the planets are moving relatively slowly in their large nearly circular orbits so that the kinetic energy available for exchange in a collision is rather small.

4. Born, Max, Atomic Physics. Authorized Translation from the German Edition by John Dougall, New York: G.E. Stechert \& Co. 1936.

5. G. Tanner, K. Richter, J.-M. Rost, Rev. Mod. Phys. 72,497 (2000).

6. Jonathan S. Parker, et al., J. Phys. B: Atomic, 33:L239 (2000).

7. R. Panfili, et al. Phys. Rev. Lett. 89, 113001, (2002).

8. G. Lagmago Kamta and A. F. Starace, Phys. Rev. A 65, 53418 (2002).

9. R. Moshamer, et al., Phys. Rev. A 65, 035401 (2002).

10. Jonathan Parker and C. R. Stroud, Jr., Phys. Rev. Lett. 56, 716 (1986).

11. A. ten Wolde et al., Phys. Rev. Lett. 61, 2099 (1988).

12. L.D. Noordam and R.R. Jones, J. Mod. Opt. 44, 2515 (1997).

13. C. Rangan, et al., J. Mod. Opt. 49, 2239 (2002).

14. Michael Nauenberg, et al., Scientific American 270, 44 (1994).

15. X. Wang and W. E. Cooke, Phys. Rev. A 46, 4347 (1992)).

16. X. Chen and J. A. Yeazell, Phys. Rev. Lett. 81, 5772 (1998).

17. J. H. Van Vleck, Proc. Nat. Acad. Sci, 14,178 (1928).

18. D. Wintgen, et al., Chaos 2, 19 (1992).

19. J. A. West, et al., Phys. Rev. A 58, 186 (1998).

20. Supported in part by the ARO-adminstered MURI Grant DAAD 19-99-1-0252.

The author is in the Institute of Optics and the Department of Physics and Astronomy at the University of Rochester, Rochester, NY 14627-0186, USA. Email:

Stroud@optics.rochester.edu. 